\title{
A Novel Function of the Receptor for Advanced Glycation End-Products (RAGE) in Association with Tumorigenesis and Tumor Differentiation of HCC
}

\author{
Kiyokazu Hiwatashi, MD, ${ }^{1}$ Shinichi Ueno, MD, PhD,${ }^{1}$ Kazuhiro Abeyama, MD, PhD, ${ }^{2}$ \\ Fumitake Kubo, MD, PhD, ${ }^{1}$ Masahiko Sakoda, MD, PhD, ${ }^{1}$ Ikuro Maruyama, MD, PhD, ${ }^{3}$ \\ Masahiro Hamanoue, MD, PhD ${ }^{4}$ Shoji Natsugoe, $\mathrm{MD}, \mathrm{PhD},{ }^{1}$ and \\ Takashi Aikou, MD, PhD ${ }^{1}$
}

\begin{abstract}
${ }^{1}$ Departments of Surgical Oncology and Digestive Surgery, Field of Oncology, Kagoshima University Graduate School of Medicine and Dental Sciences, Kagoshima, 890-8520, Japan

${ }^{2}$ Preventive Medicine funded by Shin-Nippon Biomedical Laboratories Ltd. (SNBL), Kagoshima University Graduate School of Medicine and Dental Sciences, Kagoshima, 890-8520, Japan

${ }^{3}$ Laboratory and Vascular Medicine, Kagoshima University Graduate School of Medicine and Dental Sciences, Kagoshima, 890-8520, Japan

${ }^{4}$ JA Kagoshima Kouseiren Hospital, Kagoshima, Japan
\end{abstract}

\begin{abstract}
Background: The expression of the receptor for advanced glycation end products (RAGE) has an impact on the mechanisms giving rise to characteristic features of various cancer cells. The purpose of this study was to elucidate the clinicopathological relevance of the level of RAGE expression in patients with hepatocellular carcinoma (HCC) and to explore the effect of RAGE expression on the characteristic features of HCC.

Methods: The expression of RAGE was assessed in paired cancer and noncancerous tissues with HCC, using reverse-transcription polymerase chain reaction (RT-PCR), and immunohistochemistry. The quantitative RT-PCR data were analyzed in association with the clinicopathological factors of the patients with HCC. In in vitro experiments, the survival of RAGE-transfected Cos7 and mock-transfected Cos7 cells was compared under hypoxic conditions. In addition, after reducing RAGE levels in RAGE-transfected Cos 7 cells by siRNA, similar experiments were performed.

Results: The expression of RAGE mRNA was lower in normal liver than in hepatitis and highest in HCC. Furthermore, in HCC, it was high in well- and moderately differentiated tumors but declined as tumors dedifferentiated to poorly differentiated HCC. Furthermore, HCC lines resistant to hypoxia were found to have higher levels of RAGE expression, and RAGE transfectant also showed significantly prolonged survival under hypoxia.

Conclusions: Our results suggest that HCC during the early stage of tumorigenesis with less blood supply may acquire resistance to stringent hypoxic milieu by hypoxia-induced RAGE expression.

Key Words: Hepatocellular carcinoma ( HCC ) - Receptor for advanced glycation end-products ( RAGE )-Hypoxia-Reverse-transcription polymerase chain reaction ( RT-PCR )-Immunohistochemistry.
\end{abstract}

Received August 9, 2007; accepted October 15, 2007; published online: December 15, 2007.

Grant support : Grants-in-Aid for Scientific Research from the Japan Society for the promotion of Science (no. 14370359 to SU, IM, and TA, and no.16659493 to K A).

Address correspondence and reprint requests to: Shinichi Ueno; E-mail: ueno1@m.kufm.kagoshima-u.ac.jp

Published by Springer Science+Business Media, LLC @ The Authors(s) 2007
Although many cancers arise from chronic inflammation, the relationships between carcinogenesis, cancer promotion, and its molecular characteristics remain poorly understood. Hepatocellular carcinoma (HCC), which typifies an inflammation- 
related tumor, is one of the most common malignancies in the world, especially in Asia and Africa. Japan has a high incidence of chronic viral hepatitis, cirrhosis, and HCC. The resolution of inflammatory activity at the molecular level may correlate with prevention of hepatocarcinogenesis and cancer promotion.

The receptor for advanced glycation end-products (RAGE) is a multiligand receptor classified as an immunoglobulin superfamily cell surface molecule and acts as a counter-receptor for high-mobility group box 1 (HMGB1), ${ }^{1}$ advanced glycation endproducts (AGEs), S100/calgranulins, and amyloid- $\beta$ peptides. These interactions trigger the activation of key cell signaling pathways (e.g., p38 and p44/42 MAP kinase, ${ }^{2} \mathrm{NF}-\mathrm{\kappa B}, \mathrm{cdc} 42 / \mathrm{rac},{ }^{3}$ and the generation of reactive oxygen species, and result in the production of proinflammatory cytokines. ${ }^{5}$ RAGE-mediated proinflammatory processes are now considered to contribute to the progression of many chronic diseases, such as neuropathy, nephropathy, ${ }^{6}$ macrovascular disease, amyloidoses, inflammatory conditions (e.g., rheumatoid arthritis and inflammatory bowel disease) and sepsis. ${ }^{5,7}$ In addition to RAGE-mediated proinflammatory events, recent studies have revealed that the interaction of RAGE and its ligands and the resultant signaling play a causative role in the characteristic modulation of cancer cell functions, i.e., increasing tumor invasion and metastasis. ${ }^{8}$ Furthermore, several clinical studies have demonstrated the (strong) association of RAGE expression with the malignant potential of various cancers such as gastric cancer, ${ }^{9}$ colon cancer, ${ }^{10,11}$ common bile duct cancer, ${ }^{12}$ pancreatic cancer, ${ }^{13}$ and prostate cancer, ${ }^{14}$ although one report showed a reverse correlation between RAGE expression and tumor progression. ${ }^{15}$ Thus, RAGE expression may be expected play have a significant role in the development of HCC, although no data have been reported for this tumor. In terms of HCC, it is known that the pathophysiological conditions or circumstances surrounding tumorigenesis are quite different from those reported for other cancers. For example, small HCC tumors at an early stage are continuously exposed to low oxygen and high glucose as well as the noncancerous hepatic tissues with cirrhosis in which tissue derangement occurs. ${ }^{15}$ In addition, RAGE is the receptor for AGEs produced from excessive glucose metabolism, ${ }^{16}$ extracellular HMGB1 released from necrotic cells which could be induced by hypoxia and inflammation, and serum amyloid A (SAA) produced in response to the proinflammatory cytokine IL- $6,{ }^{17}$ and these RAGE ligands are possibly generated and/ or released from inflamed hepatic tissues. Our central hypothesis is thus that RAGE expression may play a particular role in tumorigenesis of HCC, in addition to the role in the invasive and/or metastatic potential of cancer cells. With this background, the objectives of this study were: (1) to clarify the relationship between RAGE expression and the clinico-pathological features of HCC and (2) to investigate the functional role of RAGE expression in HCC development.

\section{MATERIAL AND METHODS}

\section{Human samples}

From March 2000 to September 2005, 65 patients with primary HCC were treated surgically in the Department of Surgical Oncology and Digestive Surgery, Kagoshima University School of Medicine. Of these 65 patients, 12 who had diabetes mellitus and 6 who underwent preoperative therapy were excluded from the study. RAGE expression for Diabetes patients upregulates at various tissues. ${ }^{18,19,20} \mathrm{~A}$ further 11 patients were excluded because their RNA samples were degraded. Samples from the remaining 36 patients ( 30 men and 6 women with a mean age of 67.1 years) were included in the study. Seven patients (19.4\%) were positive for hepatitis B surface antigen and $21(58.3 \%)$ were positive for the antibody to hepatitis $\mathrm{C}$ virus. Eight patients $(22.2 \%)$ were negative for both of these viruses. Twenty-four patients had chronic hepatitis and four had liver cirrhosis. The mean tumor size was $49.7 \mathrm{~mm}$ (range, $16-150 \mathrm{~mm}$ ). The histological grade of each tumor was determined according to the general rules for the clinical and pathological study of primary liver cancer (The Liver Cancer Study Group of Japan, 2000). ${ }^{21}$ Four tumors (11.1\%) were well-differentiated HCC, 28 (77.8\%) moderately differentiated HCC, and $4(11.1 \%)$ poorly differentiated HCC. Postoperative tumor recurrence was observed for 11 patients (30.6\%) (Table 1). For immunohistochemical study, a further $12 \mathrm{HCC}$ samples (six well- and six poorly differentiated HCC), obtained surgically during the same period at JA Kagoshima Kouseiren Hospital, were added and used to verify the correlation of RAGE expression with tumor differentiation. Finally, a total of 48 HCC nodules (10 well-differentiated HCC, 28 moderately differentiated $\mathrm{HCC}$, and 10 poorly differentiated HCC) were studied. As a control study, six normal liver samples were collected from patients with benign or metastatic liver tumors. Written informed consent, recognized by the ethical committees of Kagoshima 
TABLE 1. Background of patients

\begin{tabular}{ll}
\hline Gender & \\
\hline Male & $30(83 \%)$ \\
Female & $6(17 \%)$ \\
Mean age & 67.2 years \\
Virus type & \\
B & $7(19.4 \%)$ \\
C & $21(58.3 \%)$ \\
None & $8(22.2 \%)$ \\
Background of livers & \\
Chronic hepatitis & $24(60.7 \%)$ \\
Liver cirrhosis & $4(11.1 \%)$ \\
Normal liver & $8(22.2 \%)$ \\
Mean tumor size & $49.7 \mathrm{~mm}$ \\
Histological grade & \\
Well differentiated & $4(11.1 \%)$ \\
Moderately differentiated & $28(77.8 \%)$ \\
Poorly differentiated & $4(11.1 \%)$ \\
\hline
\end{tabular}

Postoperative tumor recurrence 11 (30.6\%).

University School of Medicine and JA Kagoshima Kouseiren Hospital, was obtained from each patient before tissue acquisition.

\section{Immunohistochemistry}

Consecutive 4- $\mu \mathrm{m}$ sections were cut from each paraffin-embedding block. Sections were immunostained by anti-RAGE antibody (Santa-Cruz, CA, USA) according to the conventional immunoperoxidase technique. Briefly, after peroxidase blocking with $3 \%$ $\mathrm{H}_{2} \mathrm{O}_{2} /$ methanol for $10 \mathrm{~min}$, specimens were blocked with phosphate buffered saline (PBS) containing 5\% normal horse serum (Vector Laboratories, Inc., Burlingame, CA, USA). Anti-RAGE antibody was used at $1 / 200$. After overnight incubation at $4^{\circ} \mathrm{C}$ with the primary antibody, specimens were briefly washed in PBS and incubated at room temperature with the secondary antibody conjugated with peroxidase. The specimens were then washed in PBS and color-developed by diaminobenzidine solution (DAKO). After washing with water, specimens were counterstained with Meyer's hematoxylin (Sigma Chemical Co., St Louis, MO, USA). Immunostaining of all cases was performed at one time to ensure the same conditions of antibody reaction and $\mathrm{DAB}$ exposure. A total of 48 HCC nodules (10 well-differentiated HCC, 28 moderately differentiated HCC, and 10 poorly differentiated HCC) were studied. To evaluate the immunohistochemical staining, ten fields were selected and expression in 1000 tumor cells (100 cell/fields) was evaluated with high-power $(\times 200)$ microscopy. The immunohistochemical expression of RAGE was defined as positive if distinct staining of the cell membrane was observed in at least $10 \%$ of tumor cells.

\section{Cell and cell culture}

Hepatoma cell lines HepG2, HuH7, HT17, and Li7 were kindly provided by the Cell Resource Center for Biomedical Research Institute of Development, Aging, and Cancer, Tohoku University and Hep3B was obtained from the European Collection of Cell Cultures. HepG2, Hep3B, and HT17 were cultured with Dulbecco's Modified Eagle's Medium (DMEM) $(1000 \mathrm{mg} / \mathrm{l})$ and $\mathrm{HuH} 7$ and $\mathrm{Li} 7$ were cultured with RPMI. RAGE-transfected Cos7 and its mock-transfectant were kindly provided by Drs. Yamamoto, Department of Biochemistry and Molecular Vascular Biology, Kanazawa University. These transfectants were maintained with DMEM supplemented with $10 \%$ Fetal Calf Serum (FCS) in the presence of $650 \mu \mathrm{g} / \mathrm{ml} \mathrm{G} 418$. A hypoxic environment was created by placing a cell culture dish with one pouch of Anaero Pack into an airtight jar. (Mitsubishi Gas Chemical Co., Inc., Tokyo, Japan). This created nearly $0 \% \mathrm{O}_{2}$ conditions.

\section{Gene silencing of RAGE with specific siRNA}

Cells were seeded in 12-well plates at a density of $1 \times 10^{5}$ cells per well and allowed to adhere overnight. Then siRNAs for the target gene or its control oligoribonucleotide mixed with Dharma FECT2 ${ }^{\mathrm{TM}}$ transfection reagent (Dharmacon Inc. Chicago, USA) was added to the cells and incubated for $48 \mathrm{~h}$ at $37^{\circ} \mathrm{C}$ The efficacy of gene silencing was evaluated using immunoblot analysis.

\section{MTT assay}

Cell viability was monitored after incubation for 24,36 , and 48 hours by MTT assay. Briefly, $0.5 \mathrm{mg} /$ $\mathrm{mL}$ 3-[4,5]-2,5-diphenyltetrazolium bromide (MTT) in fresh medium was added to each well and the cells were incubated for an additional 3 hours. Afterwards, the blue formazan crystals were dissolved in $1 \mathrm{~mL}$ isopropanol and measured spectrophotometrically at $570 \mathrm{~nm}$.

\section{Immunoblot analysis}

Whole cell lysates were prepared as per the Santa Cruz protocol. One milliliter of Radio-Immunoprecipitation Assay (RIPA) buffer was added to a 100 $\mathrm{mm}$ cell culture plate. The plates were gently rocked for $15 \mathrm{~min}$ at $4^{\circ} \mathrm{C}$. Adherent cells were scrpaed with a cell scraper, followed by incubation for 30-60 min on ice. The cell lysate was microcentrifuged at $10,000 \mathrm{~g}$ 
for $10 \mathrm{~min}$ at $4^{\circ} \mathrm{C}$. The supernatant fluid was the total cell lysate. The supernatant was transferred to a new microfuge tube and the pellet was discarded. Twelve microgram lysates were subjected to immunoblot analysis using a $12.5 \%$ sodium dodecyl sulfate (SDS) -polyacrylamide gel followed by electrotransfer onto nitrocellulose filters. The filters were immunoreacted with anti-RAGE antibody (a gift from TORAY Research Institute, Sagamihara, Japan) or with antiHMGB1 antibody (BD Biosciences, Tokyo, Japan) and then incubated with peroxidase-conjugated antigoat IgG (Medical and Biological Laboratories, Nagoya, Japan). The immune complex was visualized using the Enhanced Chemiluminescence (ECL) Western blot detection system (PIERCE, Rockford, IL, USA). The amount of B-actin as an internal control was also examined using a specific antibody (Cytoskelton Inc., Denver, CO, USA and Santa Cruz, CA, USA). At least three independent experiments were performed.

\section{Quantitative RT-PCR}

For reverse-transcription PCR (RT-PCR) and realtime quantitative PCR, total RNA was extracted from $30 \mathrm{mg}$ frozen tissue using Total RNA Mini (VIOGENE, CA, USA). For cDNA synthesis, the RNA samples $(1 \mu \mathrm{g})$ were converted into cDNA by reverse transcription (RT) using random primers (TAKARA, Siga, Japan) according to the manufacturer's instructions. To estimate the mRNA expression levels of several genes quantitatively, polymerase chain reaction (PCR) amplification was performed using a Light-Cycler system (Roche, Mannheim, Germany) and the Light-Cycler Fast Start DNA Master SYBER green I kit (Roche). Primers were as follows: RAGE: 5'-AAA CAT CAC AGC CCG GAT TG-3' and 5'-TCC GGC CTG TGT TCA GTT TC-3', HMGB1: 5'-GCT CAG AGA GGT GGA AGA CCA- $3^{\prime}$ and $5^{\prime}$-GGT GCA TTG GGA TCC TTG AA-3' (14), GAPDH: 5'-TTG GTA TCG TGG AAG GAC TCA- $3^{\prime}$ and $5^{\prime}$-TGT CAT CAT ATT TGG CAG GTT T-3'. Amplification was carried out in $20 \mu \mathrm{L}$ reactions containing $4 \mathrm{mM} \mathrm{MgCl}, 2 \mu \mathrm{L}$ of primer, $2 \mu \mathrm{L}$ of Light-Cycler-FastStart DNA Master SYBR green I reagent, and $2 \mu \mathrm{L}$ of cDNA. Reaction conditions were an initial incubation at $95^{\circ} \mathrm{C}$ for 10 min, followed by 50 cycles at $95^{\circ} \mathrm{C}$ for $10 \mathrm{~s}$ for denaturation, $64^{\circ} \mathrm{C}$ for $10 \mathrm{~s}$ for annealing of the RAGE primers, $54^{\circ} \mathrm{C}$ for $10 \mathrm{~s}$ for annealing of the HMGB1 primers and $60^{\circ} \mathrm{C}$ for $10 \mathrm{~s}$ for annealing of the GAPDH primers and $72^{\circ} \mathrm{C}$ for $10 \mathrm{~s}$ for extension. Melting curves were obtained according to the protocol under the following conditions: $0 \mathrm{~s}$ denaturation period at $95^{\circ} \mathrm{C}$, starting temperature of $65^{\circ} \mathrm{C}$, end temperatures of $95^{\circ} \mathrm{C}$, and rate of temperatures increase of $0.1^{\circ} \mathrm{C} / \mathrm{s}$. The quantitative value of the target gene (RAGE mRNA) in each sample was normalized using GAPDH expression as an internal control. The quantitative RT-PCR assay was carried out twice and the mean value was calculated. Finally, the mRNA expression ratio of cancerous (C) to noncancerous $(\mathrm{N})$ tissues was calculated using the following formula: $R=\log$ \{target gene (C)/GAPDH (C) $\}, R=\log \{$ target gene (N)/GAPDH (N) $\}$. These experiments were carried out twice to confirm reproducibility.

\section{Statistical analysis}

Statistical analysis was performed using the JMP IN version 5.1.2 software system (SAS institute Inc., Cary, NC, USA). Each value of mRNA expression was $\log$ transformed before statistical analysis. Gene expression was compared among normal liver, hepatitis, and HCC using Student's $t$-test. The relationships between RAGE-, HMGB1-mRNA expression levels and clinicopathological features were evaluated using Student's $t$-test and the Mann-Whitney $U$ test, as appropriate. Immunohistochemical study of RAGE in HCC tissues was evaluated using the $\chi^{2}$ test. A $p$ value of less than 0.05 was considered to be statistically significant.

\section{RESULTS}

\section{RAGE and HMGB1 expression in normal liver, hepatitis, and HCC}

RAGE antibody yielded a strong band compared to control mouse lung extracts. Using Western blotting and RT-PCR, protein and mRNA expression of RAGE and HMGB1 were examined in both cancer and noncancerous tissues from three cases (Fig. 1a, b). All 3 cases showed co-expression of RAGE and HMGB1 protein and mRNA in these tissues.

\section{Quantitative RAGE mRNA expression in HCC and noncancerous lesions}

Comparing the quantitative expression of RAGE mRNA in paired cancer and noncancerous tissues (i.e., chronic hepatitis or liver cirrhosis) of 36 cases, HCC tissues showed significantly higher expression than noncancerous tissues $(p<0.01$, Fig. 2a). 
a A
$(50 \mathrm{kd})$

Control normal $\mathrm{HCC} \quad \mathrm{CH} \quad \mathrm{HCC} \quad \mathrm{CH}$
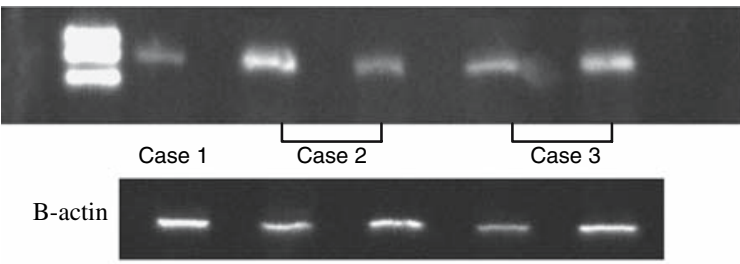

B
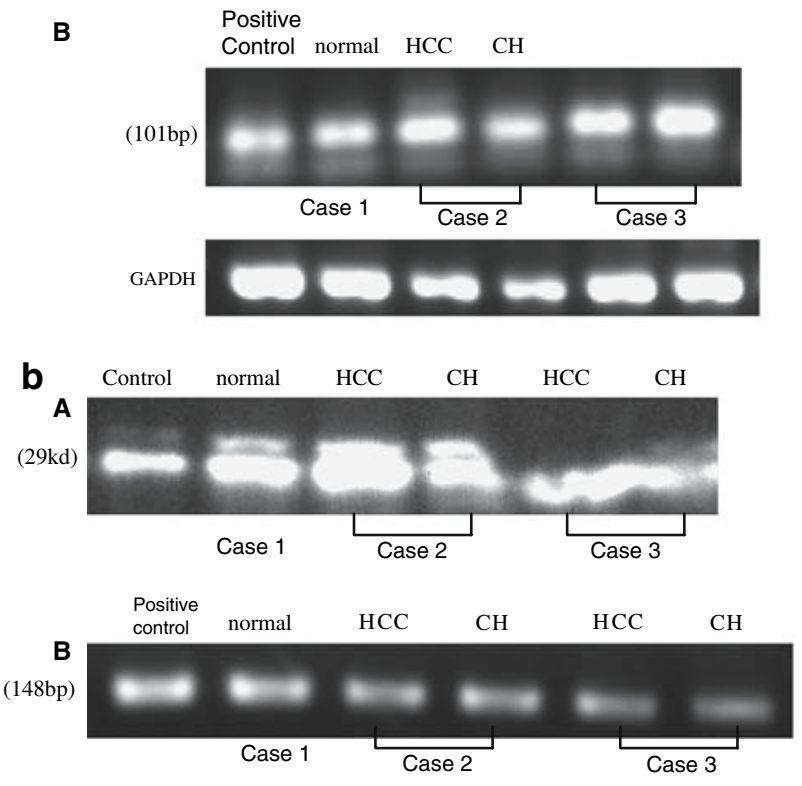

Moreover, the mean values of RAGE mRNA expression in cancer and noncancerous tissues were higher $(p<0.01$ and $p=0.08)$ than that in normal liver tissues (i.e., non-inflamed liver from benign or metastatic liver tumor patients) (Fig. 2b).

\section{Relationship between RAGE mRNA expression and clinicopathological features}

To elucidate the biological significance of RAGE expression in HCC, we compared the levels of RAGE mRNA expression with the clinico-pathological features of 36 patients. As shown in Table 2, we noted significant differences in RAGE mRNA expression in association with gender, age, the levels of protein induced by vitamin $\mathrm{K}$ absence or antagonist (PIVKA-II), and postoperative recurrence. In terms of gender and age, the results are in accordance with a previous report. $^{23}$ The expression levels of RAGE mRNA tended to be lower in poorly differentiated tumors compared to well- or moderately differentiated tumors $(p=0.06)$. Moreover, the levels of RAGE mRNA showed a negative correlation with
FIG. 1. (a) RAGE expression by Western blotting (A) and RT-PCR (B). (b) HMGB1 expression by Western blotting (A) and RT-PCR (B). Abbreviations: normal, normal liver; $\mathrm{CH}$, chronic hepatitis.

PIVKA-II levels and the presence or absence of recurrence. There were no significant differences regarding tumor size, intrahepatic metastasis, and vascular invasion. On the other hand, it should be noted that a significant difference in the levels of RAGE expression was observed between hepatitis virus-positive and virus--egative tissues $(p<0.01)$ in the study of noncancerous inflammatory liver tissues (i.e., chronic hepatitis or liver cirrhosis) (data not shown).

\section{RAGE expression and tumor differentiation in HCC}

To verify the correlation of RAGE expression with HCC differentiation, we carried out an immunohistochemical examination. (Fig. 3a-c) Well- and moderately differentiated $\mathrm{HCC}$ showed a high percentage of RAGE positivity ( $70 \%$ and $64 \%$, respectively); only 2 of $10(20 \%)$ were positive in poorly differentiated HCC (Fig. 3d). Statistically, there were significant differences in the rates of positivity between well- and moderately differentiated HCC and poorly differentiated HCC. 

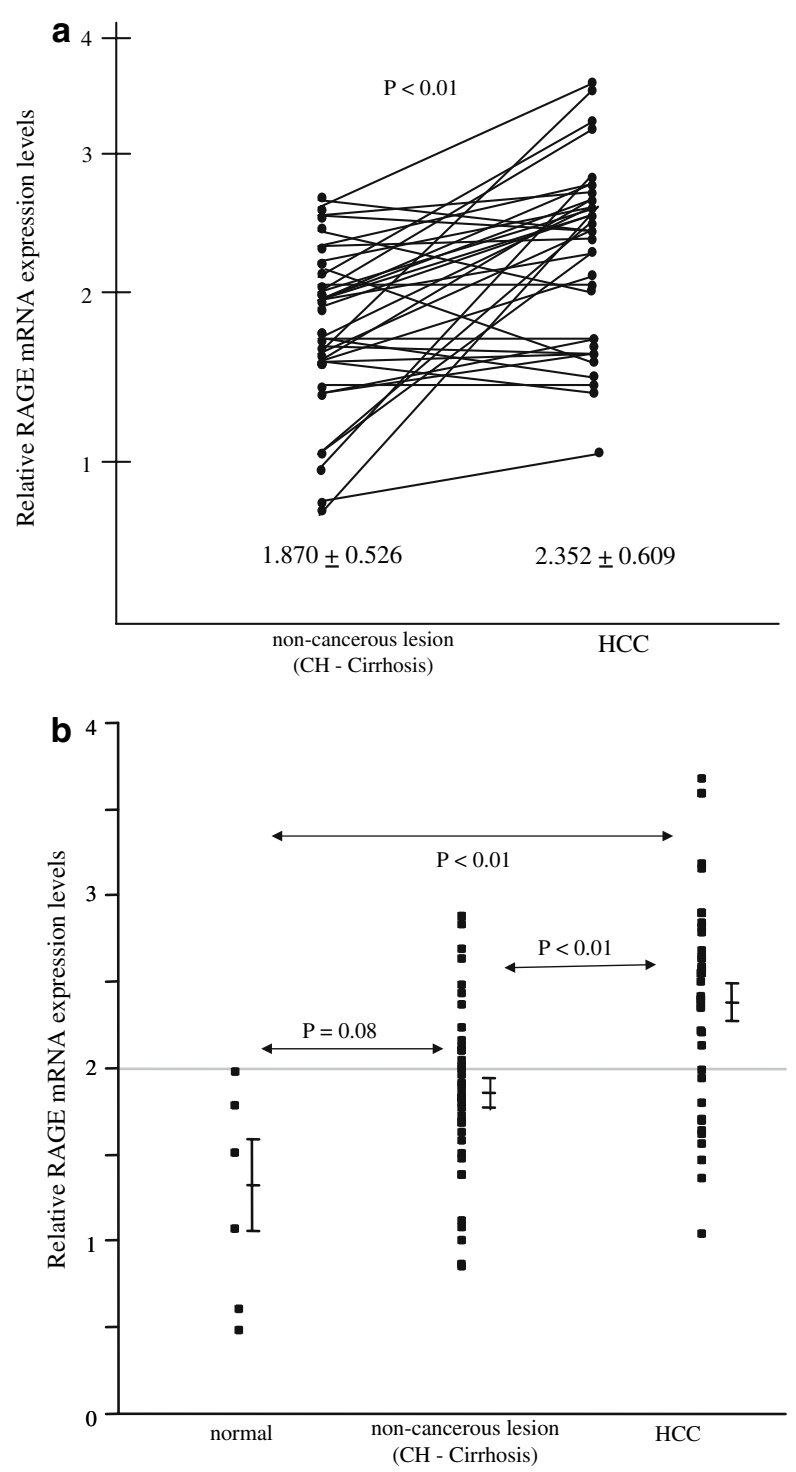

FIG. 2. (a) Quantitative RAGE mRNA expression in paired cancerous (HCC) and noncancerous tissues from 36 cases. (b) Quantitative RAGE mRNA expression in normal liver $(n=6)$ and noncancerous and cancerous tissues $(n=36)$.

\section{RAGE expression in hepatoma cell lines}

RAGE antibody yielded a strong band on Western blot analysis of the control RAGE transfected Cos7 cells (Fig. 4a, lane 1). In this experimental setting, the well-differentiated HCC cell line $\mathrm{HuH7}$ (lane 6) expressed RAGE protein at a modest level. The poorly differentiated HCC cell line HT17 (lane 7) expressed RAGE protein at a low level. Hepatoma cell lines Hep3B and Li7 (lane 4 and 5), derived from tumors of unknown state of differentiation, expressed RAGE protein at a modest level. HepG2 (lane 3) did not express RAGE protein at all. These results were very similar to RAGE mRNA expression and depend on the pathological differentiation of HCC.

\section{Enhanced RAGE expression under hypoxic conditions}

Early carcinogenesis occurs in an avascular environment and under conditions of hypoxia. ${ }^{15,23,24} \mathrm{In}$ order to clarify the role of RAGE expression under hypoxic conditions, all hepatoma cell lines were cultured in an anaerobic environment. Li7 could survive for $48 \mathrm{~h}$, Hep3B for $12 \mathrm{~h}$, and $\mathrm{HuH} 7$ for $24 \mathrm{~h}$ under such conditions, up to the appearance of signs of cell death. HepG2 and HT17 rapidly died under anaerobic conditions. The RAGE-positive cell lines (Li7, Hep3B and $\mathrm{HuH} 7$ ) were more tolerant of anaerobic conditions than RAGE-negative or weakly expressing cell lines (HepG2 and HT17). Moreover, RAGE expression in the cell lines that survived was clearly upregulated in response to the hypoxic conditions (Fig. 4b).

\section{Enhancement of cell survival in anaerobic conditions following RAGE transfection}

These findings led to the hypothesis that RAGE expression could confer tolerance to hypoxia in HCC. Therefore, we investigated the survival of RAGEtransfected and mock-transfected cells under hypoxic conditions. In continuous incubation under hypoxic conditions, as expected, RAGE-transfected Cos7 cells clearly survived longer than mock-transfected Cos7 cells (observed at $36 \mathrm{~h}$ and $48 \mathrm{~h}$ ) (Fig. 5).

\section{Decline of cell survival in hypoxic conditions following RAGE reduced by siRNA}

Consistent with the previous result, reduction of RAGE expression with anti-RAGE siRNA re-increased susceptibility to hypoxia-induced injury in RAGE-overexpressed Cos7 transfectant (Fig. 6a,b), suggesting that RAGE expression might play an important role in the acquirement of hypoxia-resistant cellular phenotype.

\section{DISCUSSION}

Hepatocellular carcinogenesis and associated tumorigenesis occur in a stringent and restrictive environment, such as the cirrhotic liver in which tissue oxygen supply is insufficient. Moreover, in the liver, with a dual blood supply from the hepatic ar- 
TABLE 2. Relationship between tumor RAGE expression and clinicopathological features

\begin{tabular}{|c|c|c|c|}
\hline \multirow[b]{2}{*}{ Factors } & \multicolumn{2}{|c|}{$\begin{array}{l}\text { Tumor RAGE mRNA } \\
\text { expression }\end{array}$} & \multirow[b]{2}{*}{$p$ value } \\
\hline & $\mathrm{n}$ & Mean \pm SD & \\
\hline \multicolumn{4}{|l|}{ Gender } \\
\hline Male & 30 & $2.267 \pm 0.341$ & \multirow[t]{2}{*}{0.03} \\
\hline female & 6 & $2.777 \pm 0.619$ & \\
\hline \multicolumn{4}{|l|}{ Age } \\
\hline$\geq 65$ years & 26 & $2.468 \pm 0.568$ & \multirow[t]{2}{*}{0.03} \\
\hline$<65$ years & 10 & $2.052 \pm 0.638$ & \\
\hline \multicolumn{4}{|l|}{ Tumor size $(\mathrm{mm})$} \\
\hline$\geq 30$ & 11 & $2.395 \pm 0.613$ & \multirow[t]{2}{*}{0.39} \\
\hline$<30$ & 25 & $2.333 \pm 0.620$ & \\
\hline \multicolumn{4}{|l|}{ Portal invasion } \\
\hline Absent & 22 & $2.358 \pm 0.655$ & \multirow[t]{2}{*}{0.52} \\
\hline Present & 14 & $2.348 \pm 0.594$ & \\
\hline \multicolumn{4}{|l|}{ Venous invasion } \\
\hline Absent & 26 & $2.339 \pm 0.577$ & \multirow[t]{2}{*}{0.47} \\
\hline Present & 10 & $2.357 \pm 0.633$ & \\
\hline \multicolumn{4}{|l|}{ Vascular invasion } \\
\hline Absent & 19 & $2.362 \pm 0.633$ & \multirow[t]{2}{*}{0.46} \\
\hline Present & 17 & $2.340 \pm 0.601$ & \\
\hline \multicolumn{4}{|c|}{ Intrahepatic metastasis } \\
\hline Absent & 27 & $2.378 \pm 0.662$ & \multirow[t]{2}{*}{0.33} \\
\hline Present & 9 & $2.273 \pm 0.435$ & \\
\hline \multicolumn{4}{|c|}{ Gross classification } \\
\hline Localized type & 21 & $2.448 \pm 0.636$ & \multirow{2}{*}{0.13} \\
\hline Invasive type & 15 & $2.217 \pm 0.564$ & \\
\hline \multicolumn{4}{|l|}{ Differentiation } \\
\hline Well & 4 & $2.365 \pm 0.566$ & \multirow{2}{*}{0.43} \\
\hline Moderately & 28 & $2.420 \pm 0.580$ & \\
\hline Poorly & 4 & $1.860+0.783$ & 0.08 \\
\hline Stage & & & \\
\hline I,II & 16 & $2.367 \pm 0.643$ & 0.55 \\
\hline III,IV & 20 & $2.340 \pm 0.597$ & \\
\hline$P I V K A \dagger U$ & & & \\
\hline Normal & 9 & $2.704 \pm 0.385$ & \\
\hline High & 25 & $2.186 \pm 0.630$ & 0.03 \\
\hline AFP & & & \\
\hline Normal & 12 & $2.557 \pm 0.586$ & 0.16 \\
\hline High & 24 & $2.250 \pm 0.607$ & \\
\hline Virus & & & \\
\hline $\mathrm{B}$ & 7 & $2.237 \pm 0.606$ & \\
\hline $\mathrm{C}$ & 21 & $2.382 \pm 0.569$ & \\
\hline None & 8 & $2.374 \pm 0.779$ & \\
\hline Recurrence & & & \\
\hline Absent & 25 & $2.485 \pm 0.628$ & 0.04 \\
\hline Present & 11 & $2.050 \pm 0.456$ & \\
\hline
\end{tabular}

tery and portal vein, liver-specific oxygen and glucose supply are available dependent upon the degree of liver disorder. The multiligand receptor RAGE is particularly relevant in this context. The ligands for RAGE may be produced abundantly during HCC development. For example, HMGB1 released from necrotic cells and AGE generated in the process of higher hepatic glucose metabolism are considered to be major candidates (i.e., the presence of HMGB1 and carboxymethyl lysine in HCC; data not shown).
Hepatic SAA production in response to IL-6 may also be included. ${ }^{19}$ In addition, RAGE expression and its downstream signaling are now considered to play a significant role in the progression of various cancers as well as the development of inflammatory lesions.

Another aspect of the biology of RAGE was suggested by the features of retinoic acid-induced neuroblastoma differentiation, in which RAGE expression played a more important role in cellular survival than in neurite outgrowth. ${ }^{25}$ In this model, inhibition of RAGE function partially blocked the increase in levels of the anti-apoptotic protein $\mathrm{Bcl}-2$ in the process of neuronal differentiation, ${ }^{25}$ indicating that RAGE and its signaling also might contribute to the survival of certain cancer cell types undergoing "differentiation". Furthermore, although indirectly, our previous study demonstrated that NF- $\kappa \mathrm{B}$, currently considered as a causative transcriptional factor for various inflammatory events and also known as a major RAGE-mediated signal, played an important role in the survival of neuronal tumor cells ${ }^{26,27}$ and in experimentally developed $\mathrm{HCC} .^{28}$

The present study demonstrated that increased RAGE expression was highly associated with the status of pathological "differentiation" in HCC, which played a significant role in acquisition of the hypoxia-resistant phenotype of tumor cells. This conclusion is supported by several lines of experimental evidence. First, the level of RAGE expression was higher in well- and moderately differentiated HCC, while it diminished as the tumors dedifferentiated to poorly differentiated HCC. This was consistent with the evidence that a negative correlation was observed between the level of RAGE mRNA expression and either the level of PIVKA II or the incidence of postoperative recurrence. Second, the analysis of five HCC lines revealed that three of these ( $\mathrm{Li} 7, \mathrm{Hep} 3 \mathrm{~B}$, and $\mathrm{HuH} 7$ ) that are resistant to hypoxic stress characteristically showed higher levels of RAGE expression compared to the two hypoxia-intolerant cell lines HepG2 and HT17. Third, sublethal hypoxia exposure induced significantly increased RAGE expression in hypoxiaresistant HCC lines. In the analysis of the association between the level of RAGE expression and the "differentiation status" of HCC lines, the level of RAGE expression was higher in "possibly differentiated" lines (i.e., HCC with low Alpha-Fet protein (AFP) production), consistent with the results from clinical samples. Finally, cells overexpressing RAGE exogenously showed prolonged survival under hypoxic conditions compared to control mock- 

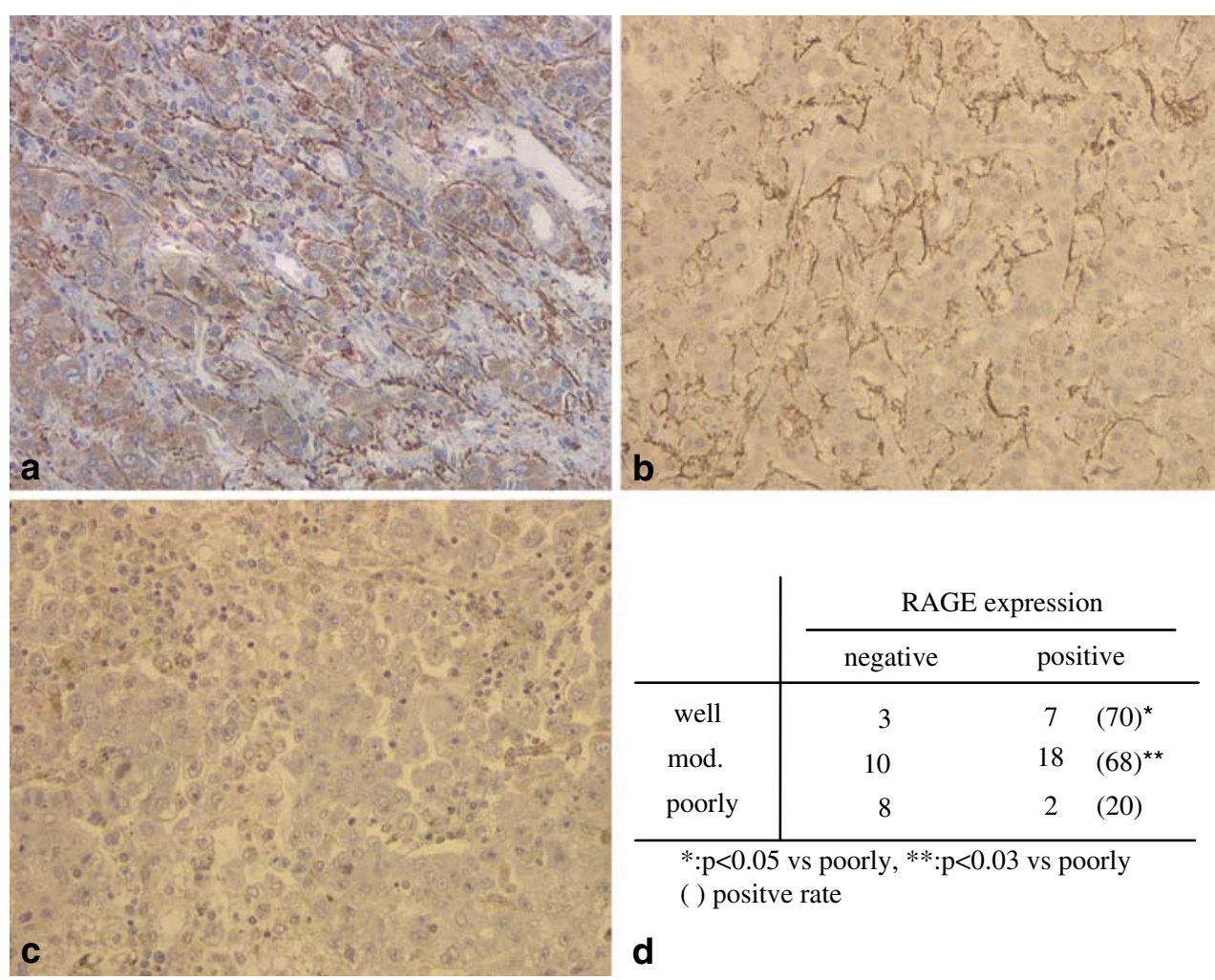

$*: \mathrm{p}<0.05$ vs poorly, ${ }^{* *}: \mathrm{p}<0.03$ vs poorly

( ) positve rate

d

FIG. 3. RAGE expression by immunohistochemical staining: (a) well-differentiated HCC, (b) moderately (mod.) differentiated HCC, (c) poorly differentiated HCC, (e) the number of RAGE positive or negative cases according to tumor differentiation.
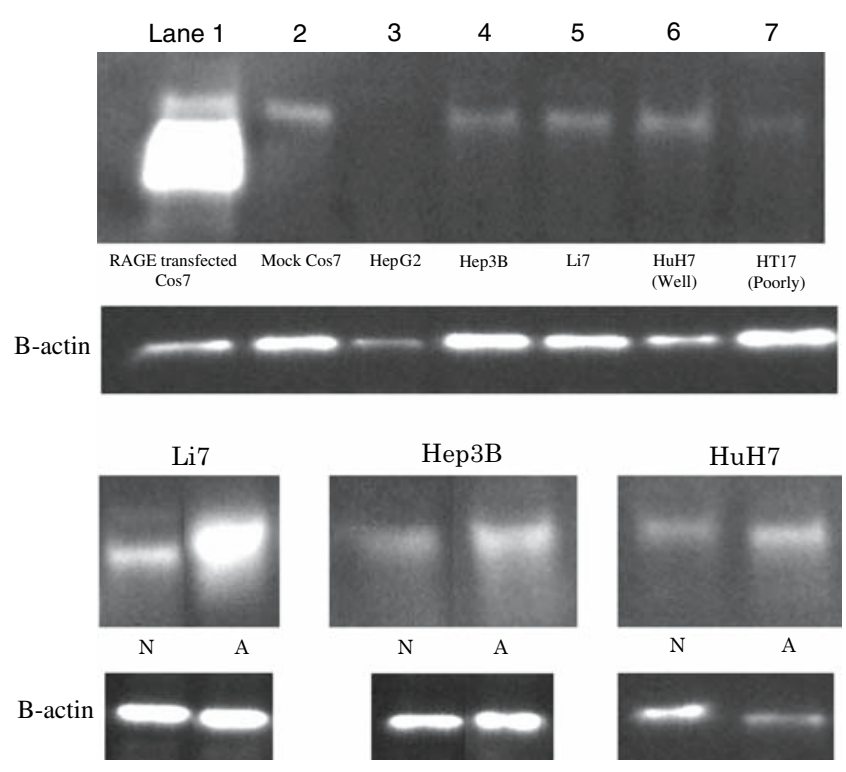

transfected cells, and siRNA experiments demonstrated similar results.

Our conclusion also provides a new hypothetical concept that hepatic RAGE expression may be
FIG. 4. (a) RAGE expression in hepatoma cell lines by Western blotting. (b) RAGE expression in hepatoma cell lines under hypoxic conditions by Western blotting. $\mathrm{N}$, normoxic conditions; A, anaerobic conditions. relevant to the stage or severity of inflammation and the incidence of carcinogenesis and early tumorigenesis of HCC (Fig. 7). In the process of the development of an HCC lesion, increased RAGE expression 


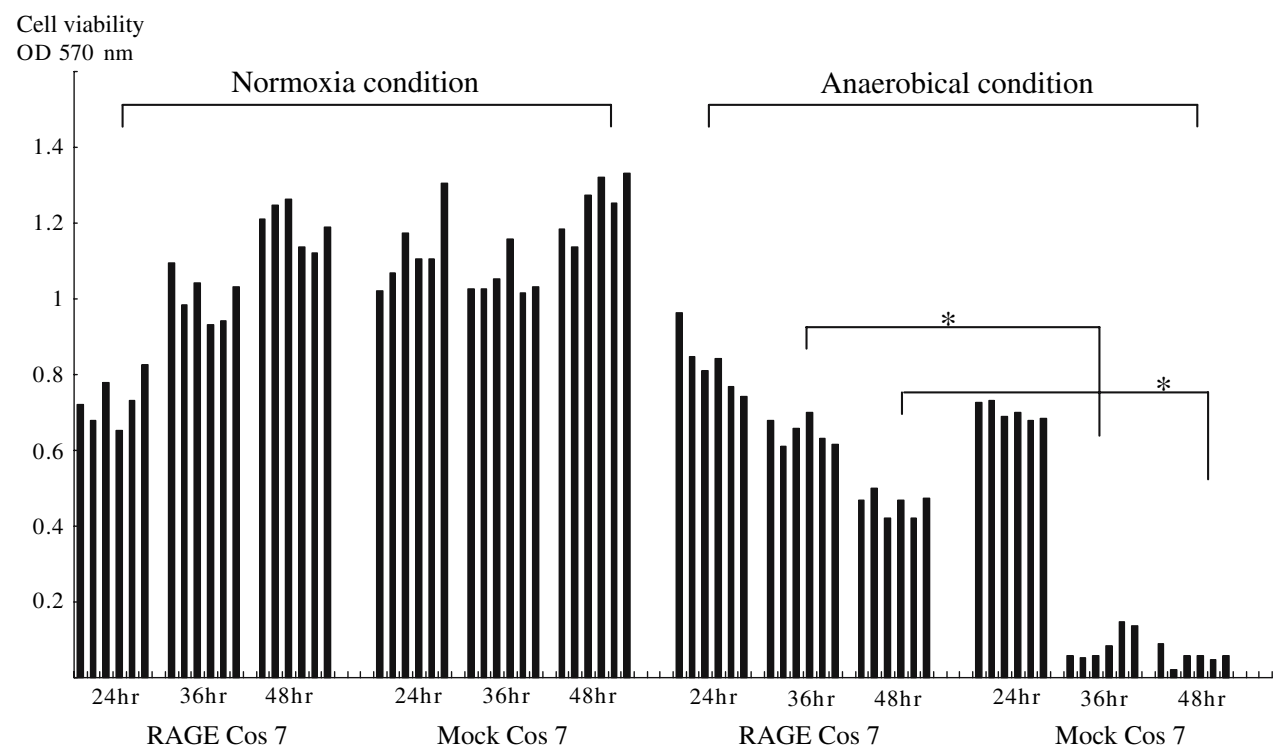

FIG. 5. Comparison of cell survival of RAGE-transfected Cos7 and mock-transfected Cos 7 cells under anaerobic conditions. Cell survival of both groups was estimated from six dishes at each time point using the MTT assay. ${ }^{*} \mathrm{p}<0.01$ by Student's t-test.

by inflamed hepatocytes may confer adaptation to an advanced hypoxic environment during remodeling and carcinogenesis with accelerated cell proliferation. In this context, an HCC lesion which is highly associated with inflammation caused by either hepatitis viruses or drugs is developed through a process of multistage carcinogenesis, i.e., from inflammatory lesions (e.g., hepatitis, cirrhosis and precancerous lesion) to adenomatous hyperplasia, and eventually into HCC. Once cancer is established, HCC dedifferentiates step-by-step to a more malignant histology, from well- and moderately to poorly differentiated HCC. It has also been pointed out that the vascular supply changes significantly between each stage of tumorigenesis. According to the evidence from several clinical studies, ${ }^{15,29,30}$ both adenomatous hyperplasia and well-differentiated HCC are hypovascular tumors primarily fed by the portal vein system, while moderately and poorly differentiated HCC are hypervascular tumors primarily fed by arterial blood. In addition, early carcinogenesis and the development of the malignant phenotype generally occur in an avascular environment. ${ }^{31-33}$ Increased RAGE expression, at least in part, may thus play an important role in the mechanism of early HCC development from precancerous inflammatory lesions. In addition, about the expression of RAGE being lower in poorly differentiated HCC when compared to well and moderately differentiated HCC, gastric and colon cancers have been showed that RAGE and other ligands interacts act on tumor promotion in clinical materials. ${ }^{9,11}$ However, prostate cancer was demonstrated to show no correlation between RAGE expression and tumor differentiation and depth. ${ }^{14}$ Moreover, non-small-cell lung cancers have been showed a reverse correlation between RAGE expression and tumor stage. ${ }^{34}$ This result is partially similar to our results. RAGE expression of each clinical samples is various.

Some reports have shown that RAGE-expressed cells have invasion and migration potential. Our data from clinical samples did no' relate to the potential. In vivo, invasive and metastatic potential are reflected by many factors, which may have caused our results.

Our results raise many questions concerning mechanistic and practical processes. It is important to know whether RAGE can bind HMGB1 secreted from activated macrophages in hepatic inflammation, or if occupancy of RAGE by inhibitors would obviate binding of the stimulatory ligands. The functional role of downstream signaling of the RAGE would also be important to determine the cytoprotective mechanism under hypoxia.

Our findings have provided the first evidence of the clinical relevance and function of RAGE in HCC, namely differentiation-associated RAGE expression that confers a hypoxia-resistant phenotype. Although other mechanisms may also be important, our data also introduce the concept that RAGE and its 


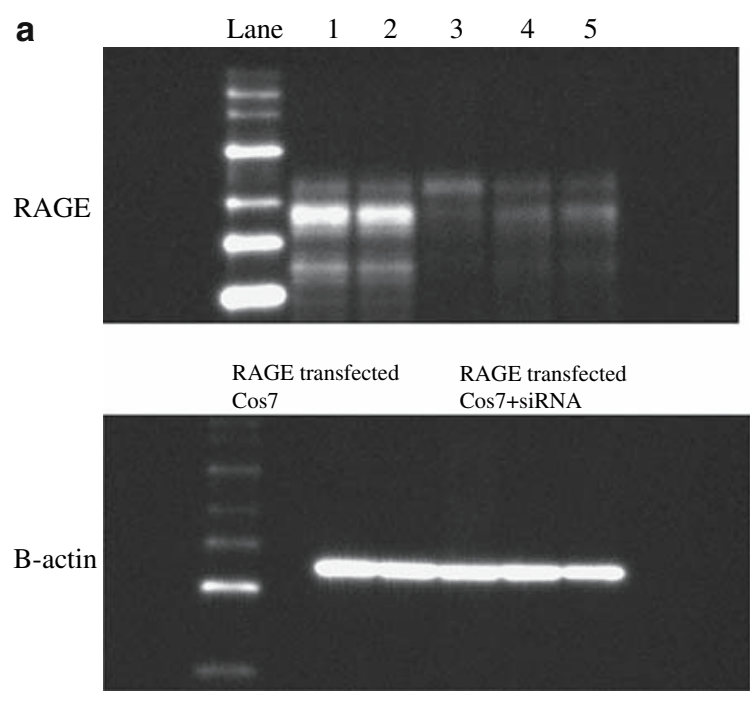

\section{b} Cell viability OD $570 \mathrm{~nm}$

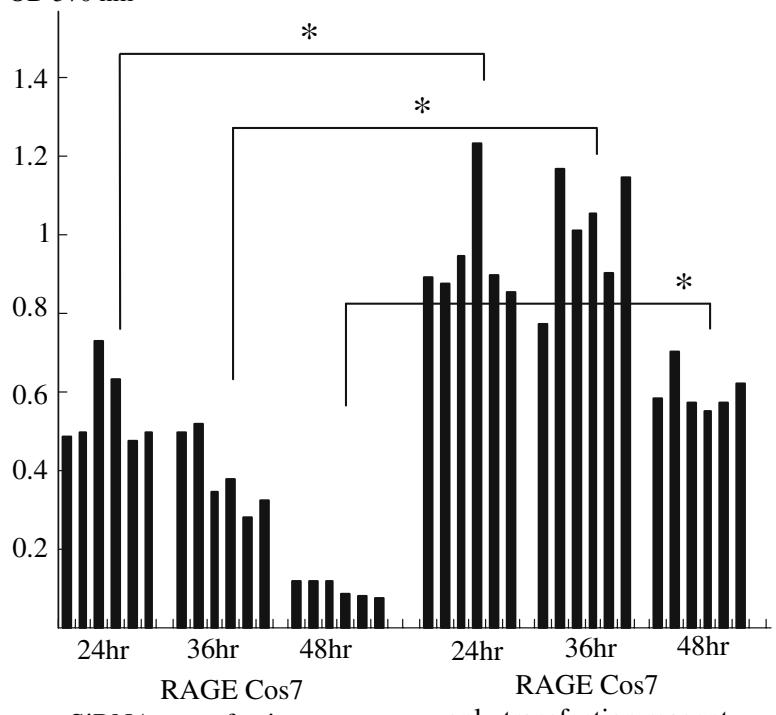

SiRNA+transfection reagent

FIG. 6. RAGE level in RAGE-transfected Cos7 cells were reduced by siRNA RAGE-transfected Cos7 cells reduced RAGE level by siRNA decreased more than the cells mixed with only transfection reagent. ${ }^{*} \mathrm{p}<0.01$ by Student's t-test.

functions may be possible candidates for therapeutic targets in the treatment of $\mathrm{HCC}$.

\section{ACKNOWLEDGEMENTS}

We thank Drs. Yasuhiko Yamamoto and Hiroshi Yamamoto (Department of Biochemistry and Molecular Vascular Biology, Kanazawa University Graduate School of Medicine) for kindly providing RAGE-transfectant and its control Cos-7 cells.

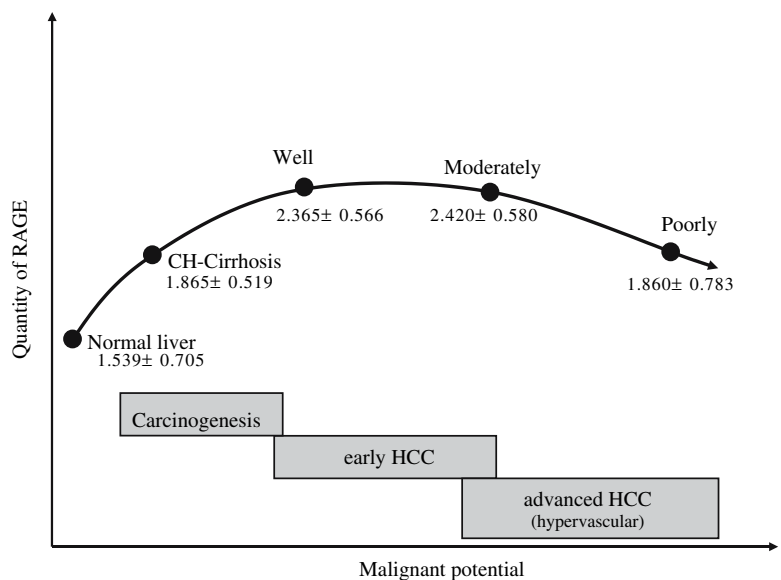

FIG. 7 Scheme of the change of RAGE expression according to the sequential change of liver tissue: normal $\rightarrow$ chronic hepatitis $(\mathrm{CH}) \rightarrow$ cirrhosis $\rightarrow$ HCC. The value indicates the quantitative RAGE mRNA expression.

\section{OPEN ACCESS}

This article is distributed under the terms of the Creative Commons Attribution Noncommercial License which permits any noncommercial use, distribution, and reproduction in any medium, provided the original author(s) and source are credited.

\section{REFERENCES}

1. Rauvala H, Pihlaskari R. Isolation and some characteristics of an adhesive factor of brain that enhances neurite outgrowth in central neurons. J Biol Chem 1987; 262:16625-35.

2. Yeh $\mathrm{CH}$, Sturgis L, Haidacher J, et al. Requirement for p38 and $\mathrm{p} 44 / \mathrm{p} 42$ mitogen-activated protein kinases in RAGEmediated nuclear factor-kappaB transcriptional activation and cytokine secretion. Diabetes 2001; 50:1495-504.

3. Huttunen HJ, Fages C, Rauvala H. Receptor for advanced glycation end products (RAGE)-mediated neurite outgrowth and activation of NF-kappaB require the cytoplasmic domain of the receptor but different downstream signaling pathways. $J$ Biol Chem 1999; 274:19919-24.

4. Wautier MP, Chappey O, Corda S, Stern DM, Schmidt AM, Wautier JL. Activation of NADPH oxidase by AGE links oxidant stress to altered gene expression via RAGE. Am $J$ Physiol Endocrinol Metab 2001; 280:E685-94.

5. Wang $\mathrm{H}$, Bloom O, Zhang M, et al. HMG-1 as a late mediator of endotoxin lethality in mice. Science 1999; 285:248-51.

6. Yamamoto Y, Kato I, Doi T, et al. Development and prevention of advanced diabetic nephropathy in RAGE-overexpressing mice. $J$ Clin Invest 2001; 108:261-8.

7. Liliensiek B, Weigand MA, Bierhaus A, et al. Receptor for advanced glycation end products (RAGE) regulates sepsis but not the adaptive immune response. J Clin Invest 2004; 113:1641-50.

8. Taguchi A, Blood DC, del Toro G, et al. Blockade of RAGEamphoterin signalling suppresses tumour growth and metastases. Nature 2000; 405:354-60.

9. Kuniyasu H, Oue N, Wakikawa A, et al. Expression of receptors for advanced glycation end-products (RAGE) is 
closely associated with the invasive and metastatic activity of gastric cancer. J Pathol 2002; 196:163-70.

10. Kuniyasu H, Chihara Y, Kondo H. Differential effects between amphoterin and advanced glycation end products on colon cancer cells. Int J Cancer 2003; 104:722-7.

11. Kuniyasu H, Chihara Y, Takahashi T. Co-expression of receptor for advanced glycation end products and the ligand amphoterin associates closely with metastasis of colorectal cancer. Oncol Rep 2003; 10:445-8.

12. Hirata K, Takada M, Suzuki Y, Kuroda Y. Expression of receptor for advanced glycation end products (RAGE) in human biliary cancer cells. Hepatogastroenterology 2003; 50:1205-7.

13. Takada M, Koizumi T, Toyama H, Suzuki Y, Kuroda Y. Differential expression of RAGE in human pancreatic carcinoma cells. Hepatogastroenterology 2001; 48:1577-8.

14. Ishiguro H, Nakaigawa N, Miyoshi Y, Fujinami K, Kubota Y, Uemura H. Receptor for advanced glycation end products (RAGE) and its ligand, amphoterin are overexpressed and associated with prostate cancer development. Prostate 2005; 64:92-100.

15. Hayashi M, Matsui O, Ueda K, et al. Correlation between the blood supply and grade of malignancy of hepatocellular nodules associated with liver cirrhosis: evaluation by CT during intraarterial injection of contrast medium. AJR 1999; 172:969-76.

16. Cooper ME. Importance of advanced glycation end products in diabetes-associated cardiovascular and renal disease. Am J Hypertens 2004; 17:31-8.

17. Yan SD, Zhu H, Zhu A, et al. Receptor-dependent cell stress and amyloid accumulation in systemic amyloidosis. Nat Med 2000; 6:633-4.

18. Chen Y, Yan SS, Colgan J, et al. Blockade of late stages of autoimmune diabetes by inhibition of the Receptor for Advanced Glycation End Products. J Immunol 2004; 173:1399405.

19. Bierhaus A, Haslbeck KM, Humpert PM, et al. Loss of pain perception in diabetes is dependent on a receptor of the immunoglobulin superfamily. J Clin Invest 2004; 114:1741-51.

20. Wendt TM, Tanji N, Guo J, et al. RAGE Drives the development of glomerulosclerosis and implicates Podocyte activation in the pathogenesis of diabetic nephropathy. Am J Pathol 2003; 162:1123-37.

21. The general rules for the clinical and pathological study of primary liver cancer. Liver Cancer Study Group of Japan. Jpn J Surg 1989;19:98-129.
22. Bierhaus A, Humpert PM, Morcos M, et al. Understanding RAGE, the receptor for advanced glycation end products. $J$ Mol Med 2005.

23. Kudo M. Morphological diagnosis of hepatocellular carcinoma: special emphasis on intranodular hemodynamic imaging. Hepatogastroenterology 1998; 45(Suppl 3):1226-31.

24. Kudo M. Imaging blood flow characteristics of hepatocellular carcinoma. Oncology 2002; 62(Suppl 1):48-56.

25. Sajithlal G, Huttunen H, Rauvala H, Munch G. Receptor for advanced end products plays a more important role in cellular survival than in neurite outgrowth during retinoic acid-induced differentiation of neuroblastoma. J Biol Chem 2002; 277:688897.

26. Abeyama K, Stern DM, Ito Y, et al. The N-terminal domain of thrombomodulin sequesters high-mobility group-B1 protein, a novel antiinflammatory mechanism. $J$ Clin Invest 2005; 115:1267-74.

27. Abeyama K, Kawano K, Nakajima T, Takasaki I, Kitajima I, Maruyama I. Interleukin 6 mediated differentiation and rescue of cell redox in $\mathrm{PC} 12$ cells exposed to ionizing radiation. FEBS Lett 1995; 364:298-300.

28. Ueno S, Aoki D, Kubo F, et al. Roxithromycin inhibits constitutive activation of nuclear factor $\{$ kappa $\}$ B by diminishing oxidative stress in a rat model of hepatocellular carcinoma. Clin Cancer Res 2005; 11:5645-50.

29. Ueda K, Terada T, Nakanuma Y, Matsui O. Vascular supply in adenomatous hyperplasia of the liver and hepatocellular carcinoma: a morphometric study. Hum Pathol 1992; 23:61926

30. Matsui O, Kadoya M, Kameyama T, et al. Benign and malignant nodules in cirrhotic livers: distinction based on blood supply. Radiology 1991; 178:493-7.

31. Gatenby RA, Gillies RJ. Why do cancers have high aerobic glycolysis?. Nat Rev Cancer 2004; 4:891-9.

32. Dewhirst MW, Secomb TW, Ong ET, Hsu R, Gross JF. Determination of local oxygen consumption rates in tumors. Cancer Res 1994; 54:3333-6.

33. Helmlinger G, Yuan F, Dellian M, Jain RK. Interstitial $\mathrm{pH}$ and $\mathrm{pO}_{2}$ gradients in solid tumors in vivo: high-resolution measurements reveal a lack of correlation. Nat Med 1997; 3:177-82.

34. Bartling B, Hofmann HS, Weigle B, Simm A. Down-regulation of the receptor for advanced glycation end-products(RAGE) supports non-small cell lung carcinoma. Carcinogenesis 2005; 26:293-301. 